\title{
Overexpression of a Gene Encoding a Catabolite Repression Element in Alternaria citri Causes Severe Symptoms of Black Rot in Citrus Fruit
}

\author{
H. Katoh, K. Ohtani, H. Yamamoto, and K. Akimitsu
}

First, second, third, and fourth authors: United Graduate School and Faculty of Agriculture, Kagawa University, Miki, Kagawa 761-0795 Japan; fourth author: PRESTO, Japan Science and Technology Agency, Kawaguchi, Saitama 332-0012, Japan. Accepted for publication 31 October 2006.

\begin{abstract}
Katoh, H., Ohtani, K., Yamamoto, H., and Akimitsu, K. 2007. Overexpression of a gene encoding a catabolite repression element in Alternaria citri causes severe symptoms of black rot in citrus fruit. Phytopathology 97:557-563.

A gene (AcCreA) encoding a catabolite repression element (CreA) with two zinc fingers of the $\mathrm{Cys}_{2} \mathrm{His}_{2}$ type was isolated from the postharvest fungal pathogen Alternaria citri. The AcCreA overexpression mutant AcOEC2 of A. citri showed normal growth on pectin medium and on segments of peel or the juice sac area from citrus fruit. Production of endopolygalacturonase, an essential virulence factor of this pathogen, was similar in AcOEC2 and the wild type in pectin-containing media. How-
\end{abstract}

ABSTRACT

Alternaria citri Ellis \& N. Pierce in N. Pierce causes the postharvest disease Alternaria black rot of a broad range of citrus fruit (4,5). A. citri infects the stem end of the fruit and causes internal black discoloration and maceration of the fruit core around the central axis area during storage $(4,5)$.

Fungal pathogens, including A. citri, produce extracellular enzymes that can degrade plant cell wall polymers during infection, and various aspects of their potential roles in pathogenicity have been examined, including penetration, maceration, nutrient acquisition, plant defense induction, and symptom expression $(7,8,42)$. In previous work, we purified endopolygaracturonases (endoPG) (EC 3.2.1.15) that can degrade pectic polysaccharide components in cell walls (16), and cloned the genes from two different species of Alternaria (17). An endoPG-disrupted mutant of A. citri had significantly reduced ability to cause black rot symptoms in citrus (17). More recently, we monitored the colonization of $A$. citri in citrus fruit tissues using mutants expressing a gene encoding green fluorescent protein (GFP) as a vital marker (18). Hyphae of the GFP transformant derived from the wild-type (G1) but not the transformant derived from its endoPG-disrupted mutant (GM4) penetrated the peel vertically, although the hyphae of both G1 and GM4 spread equally in the juice sac area when the mutants were injected directly into the central axis of fruit (18). The pattern of endoPG gene expression in various parts of citrus fruit also was examined using the $A$. citri mutant EPG7 carrying a GFP gene under the control of the 813-bp putative endoPG gene (Acpg1)

Corresponding author: K. Akimitsu; E-mail address: kazuya@ag.kagawa-u.ac.jp

The nucleotide sequence data reported in this paper appears in the DNA Data Bank of Japan, EMBL, and GenBank sequence database under accession no. AB267479 for AcCreA.

doi:10.1094/PHYTO-97-5-0557

(C) 2007 The American Phytopathological Society ever, addition of glucose to the medium showed that carbon catabolite repression of endopolygalacturonase gene (Acpg1) expression, as well as endopolygalacturonase production, was lost in AcOEC2. The wild-type strain of $A$. citri causes rot mainly in the central axis of citrus fruit without development of rotting in the juice sac area; however, AcOEC2 caused severe black rot symptoms in both the central axis and juice sac areas. These results indicate that AcCreA-mediated catabolite repression controls the virulence or infection of this pathogen, and that the wild-type A. citri does not cause symptoms in the juice sac area due to carbon catabolite repression by sugars in the juice of the juice sac area.

Additional keywords: cell wall-degrading enzyme. promoter region (18). The green fluorescence of EPG7 was induced by pectin in the peel but repressed completely in the juice sac area (18). The cause of repression in the juice sac area was expected to be carbon catabolite repression by sugars in the juice. In many organisms, glucose can repress multiple genes encoding products that metabolize carbon sources other than glucose, and several studies of yeast and filamentous fungi have revealed a putative mechanism for the glucose repression of eukaryotes that can be distinguished from the mechanism found in bacteria (30). A protein found to play roles in this carbon catabolite repression in Aspergillus nidulans was named a catabolite repression element (creA); it is a homolog of the yeast Mig1 and shares functions similar to those of Mig1 $(1,12,43)$. CreA possesses a zinc finger domain of the $\mathrm{Cys}_{2} \mathrm{His}_{2}$ type which prevents transcription of several genes in the presence of glucose by sequencespecific binding to the promoters of the target genes $(10,13,37)$. The involvement of CreA in glucose repression has been examined in several fungi $(30,32,44)$; however, the effect of carbon catabolite repression and, hence, CreA on the pathogenesis of phytopathogenic fungi is still not clear.

In this study, we examined the role of CreA in regulation of the expression of the endoPG gene, Acpgl, which is the established essential dominant virulence factor in Alternaria citri $(17,18)$, because analysis of the Acpg 1 promoter region revealed the presence of putative binding sites for CreA (26). We cloned a creA gene, designated $A c C r e A$, from $A$. citri and made an overexpression mutant with $A c C r e A$. Phenotypic examinations of the overexpression mutant showed an unexpected loss of carbon catabolite repression, and the mutant gained the ability to cause rotting in the juice sac area of citrus fruit.

\section{MATERIALS AND METHODS}

Fungal culture conditions and measurement of endoPG activity. Mycelial fragments of A. citri (American Type Culture 
Collection 58171) (9) and the CreA overexpression mutant AcOEC2 were stored in $25 \%$ (vol/vol) glycerol at $-80^{\circ} \mathrm{C}$ and were grown on V8 agar plates immediately before use in various experiments. They were grown in potato dextrose broth (PDB) or in liquid pectin or pectin agar medium containing $1 \%(\mathrm{wt} / \mathrm{vol})$ pectin (P-9135; Sigma-Aldrich, St. Louis) with or without addition of $2 \%(\mathrm{wt} / \mathrm{vol})$ glucose, as described previously (16-18). The fungi were grown in 200-ml flasks each containing $50 \mathrm{ml}$ of pectin liquid medium for 10 or 25 days at $24^{\circ} \mathrm{C}$, the culture filtrates were filtered through four layers of gauze for endoPG enzyme assays, and the mycelia mats were recovered for isolation of nucleic acids. EndoPG activity of the cultures was measured by a relative viscosity assay, and one unit was defined as the activity that reduces the viscosity of a $1.5 \%$ polygalacturonic acid solution (pH 5.0) at $37^{\circ} \mathrm{C}$ in $5 \mathrm{~min}$ to $50 \%$, as described previously (16).

Isolation of the $A$. citri gene encoding CreA. Genomic DNA from wild-type $A$. citri was isolated from the mycelial mats grown in PDB by the method of Isshiki et al. (17). Total RNA was isolated from mycelial mats grown at $24^{\circ} \mathrm{C}$ for 14 days in PDB or for 10 or 25 days in pectin liquid medium, according to the method of Masunaka et al. (23).

A pair of polymerase chain reaction (PCR) primers, CREAF5 (5'-GARAARCCNCAYGCNTG-3', forward primer) and CREAR5 (5'-GTRTGRTCNGGNGTNGG-3', reverse primer) (Table 1), designed from the conserved region of deduced amino acid sequences of CreAs from several fungal phytopathogens, was used for PCR amplification of a portion of the A. citri gene encoding CreA. PCR was performed in a Gene Amp PCR System 9700 (Applied Biosystems, Foster City, CA) with Taq DNA polymerase (2.5 units; Takara, Shiga, Japan) at $95^{\circ} \mathrm{C}$ for 2 min for one cycle, and 30 cycles of $2 \mathrm{~min}$ at $95^{\circ} \mathrm{C}, 2 \mathrm{~min}$ at $50^{\circ} \mathrm{C}$, and $1 \mathrm{~min}$ at $72^{\circ} \mathrm{C}$, with $2 \mu \mathrm{M}$ CREAF5 and CREAR5, $2.5 \mathrm{mM}$ each deoxynucleotide triphosphate (dNTP), 100 ng of genomic DNA from A. citri, and the reaction buffer supplied with the Taq DNA polymerase (Takara).

Rapid amplification of cDNA ends (RACE) was used to obtain the full-length cDNAs using a Marathon cDNA Amplification Kit (Clontech, Palo Alto, CA). Total RNA (6 $\mu \mathrm{g})$ was reverse transcribed with an oligo (dT) primer, and second-strand cDNA synthesis and ligation of adaptors was performed with the Marathon kit. Subsequent PCR was conducted with the Clontech Adapter Primer 1 (AP1) (5'-CCATCCTAATACGACTCACTATAGGGC-3') (Table 1) and either forward primer CREAF5 (3' RACE) or reverse primer CREAR5 (5' RACE). The products were subcloned into a pTBlue-2 T-vector (Novagen, Madison, WI). The sequences of $5^{\prime}$ and $3^{\prime}$ RACE products were obtained from both strands by the dideoxy chain termination method (33) with the use of an ABI PRISM Dye Termination Cycle Sequencing Ready Reaction Kit (Applied Biosystems) and an automated fluorescent DNA sequencer (Model 310; Applied Biosystems). Another set of PCR primers, UCREAF1 (forward primer, 5'-ACAGCGGGGACCCTCGGTCAC-3') (Table 1) and UCREAR3 (reverse primer, 5'-AG-
TTTGTCCATATCTAATCTCATCGCTTGC-3') (Table 1), designed from the upper end of the $5^{\prime}$ RACE product and the untranslated region (UTR) downstream from the $3^{\prime}$ RACE product was used to amplify the entire region of the creA gene from either genomic DNA or cDNA, under the PCR conditions as described for the amplification of the internal region above, except that the concentration of primers UCREAF1 and UCREAR3 was $1 \mu \mathrm{M}$ each. DNA sequences were aligned with the CLUSTAL W program (36), and sequence analysis was performed using the PROSITE program (15), PSOERT program (25), SMART program (34), and GENETYX-MAC (Software Development Ltd., Tokyo). Homology analysis was performed using BLAST at the DNA Data Bank of Japan.

Blotting conditions. For Southern blot analysis, a PCR digoxigenin (DIG) probe was prepared from the AcCreA sequence in a pTBlue-2 T-vector as a template for labeling using the PCR DIG Probe Synthesis Kit (Roche, Mannheim, Germany) with primers CREAF5 (forward primer) and CREAR5 (reverse primer), following the manufacturer's instructions. For northern blot analysis, another PCR DIG probe for the entire coding region of AcCreA also was prepared with primers CreAF (ATG) (5'ATGCAATCCAACTCTGCCTC-3') and CreAR (withoutTAA) (5'-GTTGCGATGTGACAGGTCCT-3') and an Acpgl PCR probe with PG\#6 (5'-ACAATGGTTGCCTTAACT-3') and PG\#8 (5'-ACGAACACTTGGTAACGC-3') (17), following the manufacturer's instructions.

Genomic DNA $(5 \mu \mathrm{g})$ was digested with 10 units of restriction enzyme EcoRI, SacI, or HindIII according to the manufacturer's instructions (Takara) and fractionated in 1\% agarose gels. Total RNA was extracted using the RNeasy Plant Mini Kit (Qiagen, Valencia, CA) from mycelial mats harvested after 10 or 25 days of incubation. Total RNA was loaded on $1 \%$ agarose-formaldehyde gels ( 5 or $10 \mu \mathrm{g} / \mathrm{lane}$ ) and transferred to a Hybond $\mathrm{N}^{+}$membrane (GE Healthcare Bio-Sciences, NJ) by capillary transfer. Total RNA on the membrane was stained with $0.02 \%$ (wt/vol) methylene blue in $0.3 \mathrm{M}$ sodium acetate $(\mathrm{pH}$ 5.2) and destained in $20 \%$ ( vol/vol) ethanol. The rRNAs were stained with methylene blue to confirm equal RNA loading. Hybridization was performed at $68^{\circ} \mathrm{C}$ overnight with the PCR DIG probe $(100 \mathrm{ng} / \mathrm{ml})$ for both Southern and northern blots, and was visualized with anti-DIG antibody-alkaline phosphatase and CSPD and exposing the membranes to X-ray films (Fujifilm RX-U, Tokyo).

Preparations of plasmid construct and $\mathrm{AcCreA}$ overexpression mutant. The overexpression vector pAOEIK was produced by ligation of the cassette of a $\operatorname{trp} C$ promoter (20) and trpC terminator (24) of Aspergillus nidulans, which were recovered from pSH75 (19), into pCR-script SK Amp (+) (Stratagene, La Jolla, CA) at BamHI and XhoI sites. A full-length AcCreA sequence was amplified with the primer set EcoRIsite+ CreAF (5'-GGAATTCCATGCAATCCAACTCTGCCTCGACAGGT-3') and CreAR+EcoRI3 (5'-CCGGAATTCTTAGTTGCGATGTGACAGGTC-3') (Table 1). Insertion of the amplified

TABLE 1. Primers used in this study

\begin{tabular}{llll}
\hline Primer name & \multicolumn{1}{c}{ Sequence $\left(5^{\prime}\right.$ to $\left.3^{\prime}\right)$} & \multicolumn{1}{c}{ Purpose $^{\mathrm{a}}$} & \multicolumn{1}{c}{ Reference } \\
\hline CREAF5 & GARAARCCNCAYGCNTG & Degenerate PCR, RACE, probe preparation & This study \\
CREAR5 & GTRTGRTCNGGNGTNGG & Degenerate PCR, RACE, probe preparation & This study \\
AP1 & CCATCCTAATACGACTCACTATAGGGC & RACE & Clontech kit \\
UCREAF1 & ACAGCGGGGACCCTCGGTCAC & Amplification of full-length AcCreA & This study \\
UCREAR3 & AGTTTGTCCATATCTAATCTCATCGCTTGC & Amplification of full-length AcCreA & This study \\
EcoRIsite+CreAF & GGAATTCCATGCAATCCAACTCTGCCTCGACAGGT & Subcloning to pAOEIK & This study \\
CreAR+EcoRI3 & CCGGAATTCTTAGTTGCGATGTGACAGGTC & Subcloning to pAOEIK/insertion check & This study \\
PtrpCF2 & GTCATACCTTCTTAAGTTCGCC & Insertion check & This study \\
CreAF (ATG) & ATGCAATCCAACTCTGCCTC & Probe preparation & This study \\
CreAR (without TAA) & GTTGCGATGTGACAGGTCCT & Probe preparation & This study \\
PG\#6 & ACAATGGTTGCCTTAACT & Probe preparation & Isshiki et al. 2001 (17) \\
PG\#8 & ACGAACACTTGGTAACGC & Probe preparation & Isshiki et al. 2001 (17) \\
\hline
\end{tabular}

${ }^{\mathrm{a}} \mathrm{PCR}=$ polymerase chain reaction and $\mathrm{RACE}=$ rapid amplification of cDNA ends . 
AcCreA at the EcoRI site in the overexpression vector, resulting in the construction of pAOEIK-AcCreA, was confirmed by PCR analysis with primer set PtrpCF2 (5'-GTCATACCTTCTTAAGTTCGCC-3') (Table 1) and CreAR+EcoRI3 and sequencing of the product.

Protoplasts of Alternaria citri were prepared with Lysing enzyme (Sigma-Aldrich) at $10 \mathrm{mg} / \mathrm{ml}$ and Kitalase (Wako, Tokyo) at $30 \mathrm{mg} / \mathrm{ml}$ as described previously (17). Co-transformation was performed with $\approx 10^{7}$ protoplasts and $3 \mu \mathrm{g}$ each of pAOEIKAcCreA and pSH75 (19), which contains a cassette conferring hygromycin resistance. Following the transformation, protoplasts were placed directly onto the regeneration medium containing $0.1 \%$ (wt/vol) Bacto yeast extract, $0.1 \%$ (wt/vol) Bacto casein digest, $1 \mathrm{M}$ sucrose, and $0.8 \%$ (wt/vol) Bacto agar and incubated at $24^{\circ} \mathrm{C}$ for $24 \mathrm{~h}$. The plates were overlaid with $5 \mathrm{ml}$ of the same medium containing $1.5 \%$ (wt/vol) Bacto agar and hygromycin B at $100 \mu \mathrm{g} / \mathrm{ml}$ and were incubated at $24^{\circ} \mathrm{C}$ for 1 day. The colonies growing on the selective medium were moved to potato dextrose agar containing hygromycin B at $50 \mu \mathrm{g} / \mathrm{ml}$ and purified by singlespore isolation. Ten transformants were obtained. Insertion of the construct was confirmed by PCR of genomic DNA with the primer set PtrpCF2 and CreAR+EcoRI3 or by northern blotting using an AcCreA PCR probe.

Colonization and pathogenicity assays. For examination of colonization of the AcCreA overexpression mutant of A. citri, a piece $\left(3 \mathrm{~mm}^{3}\right)$ of mycelium growing on a V8-medium agar plate was placed on the internal surface of peel segments $(2$ by $2 \mathrm{~cm})$ or juice sac segments $(2$ by $2 \mathrm{~cm}$ ) from a Washington navel orange (Citrus sinensis (L.) Osbeck). The peel or juice sac segments had been presterilized (17) by soaking for $1 \mathrm{~h}$ in $1 \%$ (vol/vol) sodium hypochlorite and then rinsed in sterile water several times. The segments were incubated in sealed sterilized plastic plates at $24^{\circ} \mathrm{C}$ for 5 days, and mycelial growth was monitored daily. The inoculation assay was independently repeated three times.

A spore suspension $(100 \mu \mathrm{l})$ containing $10^{5}$ spores of either the wild-type A. citri or AcCreA overexpression mutant AcOEC2 was injected directly into the central axis of sterilized citrus fruit in accordance with the method of Isshiki et al. $(17,18)$. Citrus cultivars such as the Washington navel orange $(C$. sinensis) and Hassaku orange ( $C$. hassaku) were used for these inoculation assays. Citrus fruit were sterilized by soaking 1 to $3 \mathrm{~h}$ in $1 \%$ ( vol/vol) sodium hypochlorite and then rinsed in sterilized water. The inoculated fruit were incubated in a sealed, sterilized plastic box at $24^{\circ} \mathrm{C}$ for 2 or 4 weeks, and the fruit was sliced in half for observation of symptom development. The inoculation assay was repeated independently six times for each cultivar.

\section{RESULTS}

Isolation of $\boldsymbol{c r e A}$ from $\boldsymbol{A}$. citri. The deduced amino acid sequences of creA from Aspergillus nidulans (12) (databank accession no. L03563), Botrytis cinerea (39) (accession no. Y16625), Cochliobolus carbonum (37) (accession no. AF306571), Gibberella fujikuroi (39) (accession no. Y16626), and Sclerotinia sclerotiorum (40) (accession no. AJ000976) were aligned, and degenerate PCR primers were designed from the conserved regions of these fungal creA sequences (Fig. 1). PCR amplification using primers CREAF5 (forward primer) and CREAR5 (reverse primer) (Table 1) and genomic DNA of Alternaria citri as a template gave a 703-bp product. The nucleotide sequence of the product showed a high degree of sequence similarity to known fungal creA genes. RACE was used to clone the full-length gene using total RNA of $A$. citri grown on pectin liquid medium containing 2\% (wt/vol) glucose. 5' RACE with the CREAR5 amplified a 946-bp product, and the CREAF5 primer amplified a 1,447-bp 3' RACE product. From the sequences of the RACE products, two additional gene-specific primers, UCREAF1 and UCREAR3 (Table 1), each encompassing the 5' and 3' UTRs of the gene, were designed. PCR amplification with these primers and sequencing the product confirmed the full sequence of $A$. citri creA cDNA, designated AcCreA (databank accession no. AB267479). This cDNA consists of 1,346 bp containing a 1,290bp open reading frame flanked by a $21-b p 5^{\prime}$ UTR sequence and a 35-bp 3' UTR. The primer UCREAF1 and UCREAR3 (Table 1) amplified a 1,395-bp product from genomic DNA, and comparison of the two sequences from cDNA and genomic DNA identified a 49-bp intron at nucleotide position 339 to 387 in the genomic sequence. Except for the intron region, the sequences of PCR products using the primers described above from both total RNA and genomic DNA were identical.

The creA gene of $A$. citri consisted of a 1,290-bp open reading frame encoding a predicted protein product of 430 amino acids (Fig. 1) with an estimated molecular mass of $46.4 \mathrm{kDa}$ and a $\mathrm{pI}$ of 9.59. CLUSTAL W analysis showed 57, 52, 92, 52, and 50\% amino acid sequence identity with the sequences of CreA from Aspergillus nidulans (12) (databank accession no. L03563),

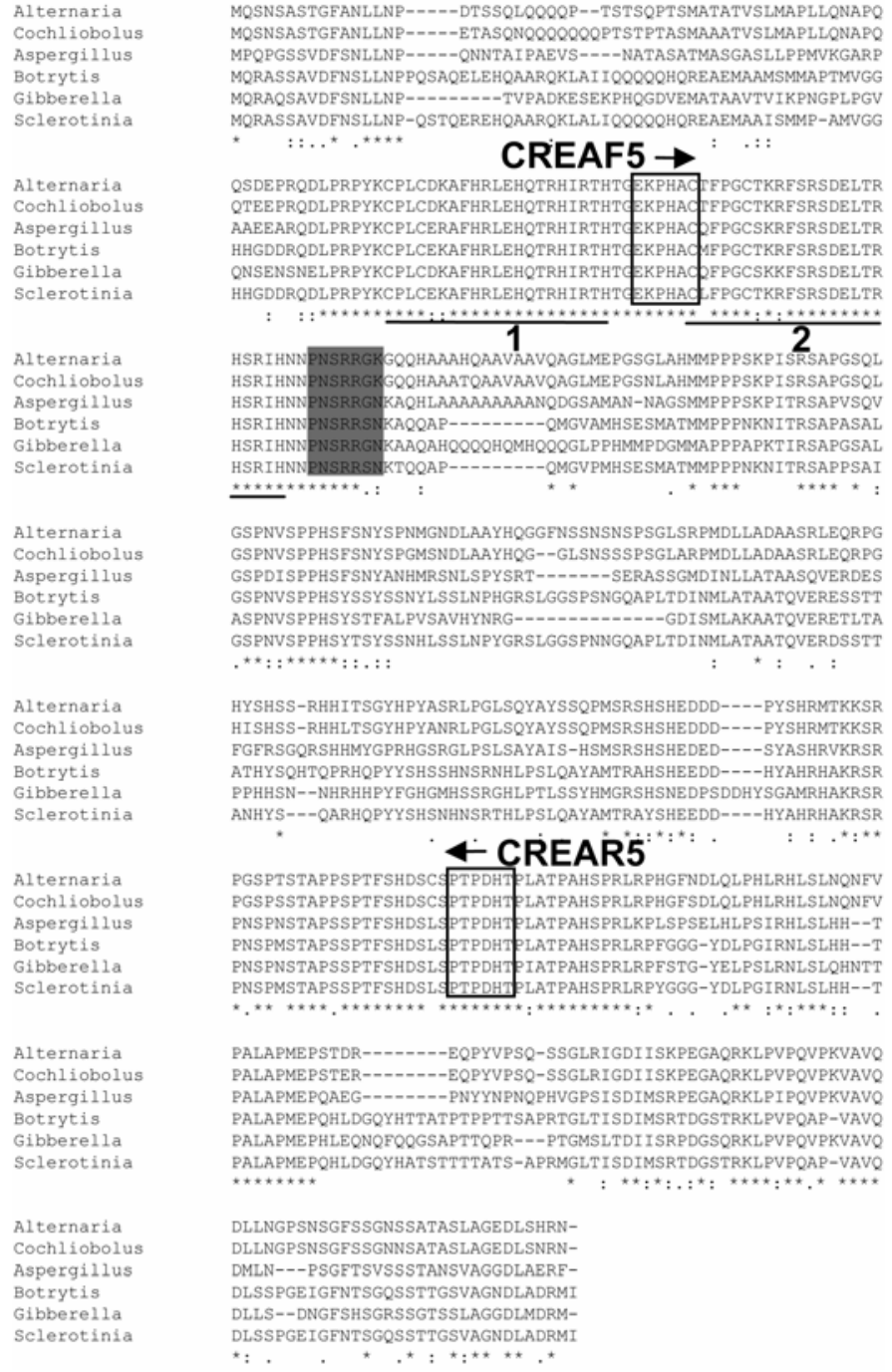

Fig. 1. Comparison of deduced amino acid sequences of AcCreA from Alternaria citri and other fungal CreA proteins. CreA sequences from phytopathogenic fungi were aligned by CLUSTAL W (36), and identical residues are indicated with asterisks. The two $\mathrm{Cys}_{2} \mathrm{His}_{2}$ zinc finger regions $(3,31)$ are underlined with numbers 1 and 2 . Two boxes with arrows indicate the annealing regions for degenerate primers CREAF5 and CREAR5 and direction of extension. The gray box indicates a putative nuclear localization signal (14). Databank accession numbers for CreAs are AB267479 for A. citri (this study), AF306571 for Cochliobolus carbonum (37), L03563 for Aspergillus nidulans (12), Y16625 for Botrytis cinerea (39), Y16626 for Gibberella fujikuroi (39), and AJ000976 for Sclerotinia sclerotiorum (41). 
B. cinerea (39) (accession no. Y16625), C. carbonum (37) (accession no. AF306571), G. fujikuroi (39) (accession no. Y16626), and S. sclerotiorum (40) (accession no. AJ000976), respectively (Fig. 1). Sequence analysis using the PROSITE program (15) identified two $\mathrm{Cys}_{2} \mathrm{His}_{2}$-type zinc fingers at amino acid positions 68 to 88 and 96 to 118 , indicating that this protein has a putative DNA binding function (Fig. 1). AcCreA also possessed a nuclear localization signal (14) of PNSRRGK, at amino acid position 121 to 127 (Fig. 1).

Hybridization of a probe corresponding to the internal part of the AcCreA gene using genomic DNA of Alternaria citri digested with EcoRI, SacI, or HindIII resulted in the appearance a single band for the EcoRI-digested genome and several additional bands in the other two enzyme-digested genomes (Fig. 2A). These restriction enzymes were used because the sequence of the cloned AcCreA gene does not have sites for EcoRI, SacI, or HindIII.

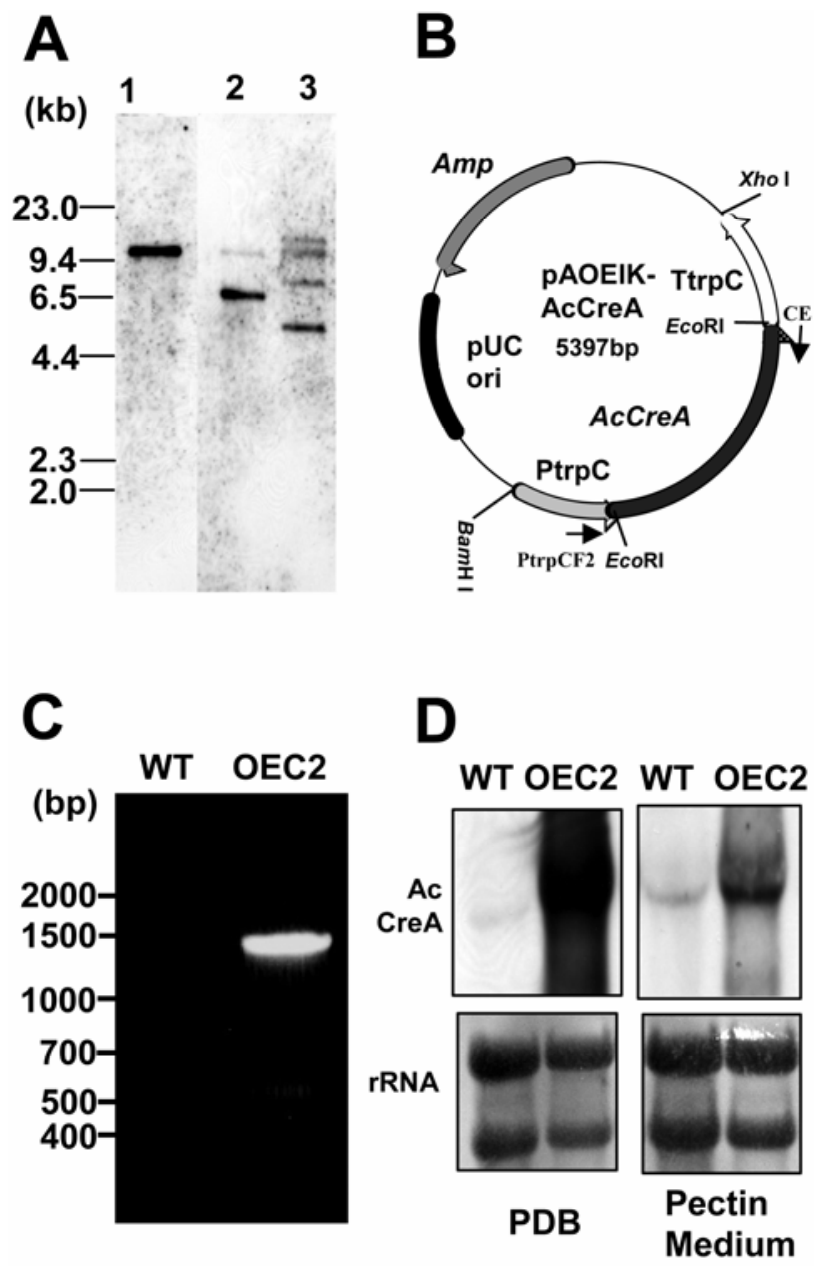

Fig. 2. Construction of an overexpression mutant of AcCreA from Alternaria citri. A, Examination of copy number of the AcCreA gene in the A. citri genome. Southern blot analysis of total genomic DNA from A. citri digested with EcoRI (lane 1), SacI (lane 2), or HindIII (lane 3) using a AcCreA-specific probe for the internal region of this gene. B, Map of plasmid construct of AcCreA overexpression vector pAOEIK-AcCreA. Arrows indicate the annealing sites for polymerase chain reaction (PCR) primers of PtrpCF2 and CreAR+EcoRI3 (CE). C, PCR analysis of insertion of AcCreA overexpression vector pAOEIK-AcCreA in the A. citri genome. Genomic DNA from wildtype A. citri (WT) or AcCreA overexpression mutant AcOEC2 (OEC2) was used for PCR amplification with primer sets of PtrpCF2 and CreAR+EcoRI3 (panel B) for detection of pAOEIK-AcCreA insertion. D, Confirmation of overexpression of AcCreA in AcOEC2 mutant. Northern blot analysis of the AcCreA gene was performed with total RNAs isolated from mycelial mats of wild-type A. citri (WT) or of the AcCreA overexpression mutant AcOEC2 (OEC2), from 10-day-old cultures in potato dextrose broth (PDB), or from 25-day-old cultures in pectin medium. Equality of RNA loading in each lane was estimated by rRNA stained with methylene blue.
Construction of CreA overexpression mutants. Hygromycinresistant transformants of $A$. citri were generated by cotransformation with the vector pSH75 and AcCreA overexpression vector pAOEIK-AcCreA (Fig. 2B). The overexpression vector pAOEIK was constructed in a PCR-Script SK $(+)$ vector (Stratagene) with additions of the constitutive expression $\operatorname{trp} C$ promoter (20) and $\operatorname{trp} C$ terminator (24) of Aspergillus nidulans. Integration of pAOEIK-AcCreA into the Alternaria citri genome was confirmed by detection of a band of the expected size of 1,430 bp by PCR analysis (Fig. 2C).

To confirm overexpression of the AcCreA gene, AcCreA transcripts were detected by northern blot analysis. Northern blots identified transcripts with the expected size of $\approx 1,300 \mathrm{bp}$ in the total RNA from AcOEC2 grown in both PDB and pectin media, whereas only traces of the transcripts were detected in total RNA of the wild type grown in PDB or pectin medium (Fig. 2D).

Characterization of CreA overexpression mutants. The CreA overexpression mutant AcOEC2 was able to utilize pectin as a sole carbon source for growth in a liquid medium. A block of AcOEC2 or wild-type A. citri mycelia was placed on the pectin medium with a sterilized piece of the internal surface of citrus peel or the citrus juice sac area. The growth of this mutant on pectin medium was slightly slower than that of the wild type, and the growth on citrus peel and citrus juice sac area of AcOEC2 was similar to that of the wild-type strain (Fig. 3A). EndoPG activity measured by the decrease in relative viscosity of a $1.5 \%(\mathrm{wt} / \mathrm{vol})$ solution ( $\mathrm{pH}$ 5.0) of polygalacturonic acid also showed normal endoPG production in the culture filtrates from the AcCreA overexpression mutant AcOEC2 similar to that of the wild type (Fig. 3B). AcOEC2 sporulated as well as the corresponding wild types, and there were no differences in spore germination, appressorium formation, or infection hypha formation on cellulose membranes (data not shown). Of 10 transformants obtained, 3 were examined in the same manner and all showed similar results, and one of the three transformants, AcOEC2, was further examined.

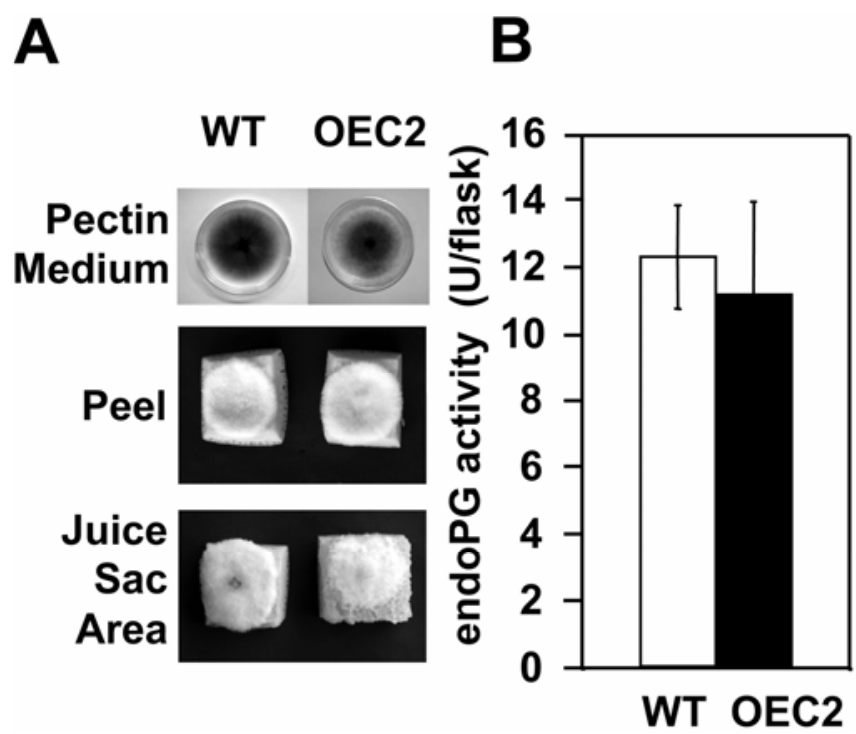

Fig. 3. Growth and endopolygaracturonase (endoPG) production by overexpression mutant AcOEC 2 in pectin medium and plant materials. A, Block of mycelia of wild-type Alternaria citri (WT) or AcCreA overexpression mutant AcOEC2 (OEC2) was placed on pectin medium and plant materials (Peel or Juice sac area). Typical growth after a 5-day incubation is shown. B, EndoPG production by $A$. citri (WT) or AcCreA overexpression mutant AcOEC2 (OEC2). Total enzyme activity produced by either fungus in $50 \mathrm{ml}$ of pectin medium after a 25 -day incubation at $24^{\circ} \mathrm{C}$ was measured by a relative viscosity assay. One unit was defined as the activity that reduces $50 \%$ of viscosity of a $1.5 \%$ polygalacturonic acid solution $(\mathrm{pH} 5.0)$ at $37^{\circ} \mathrm{C}$ for $5 \mathrm{~min}$, as described previously (16). Data indicate the mean of five independent repeats with standard deviation values. 
EndoPG gene (Acpg1) expression and endoPG production were examined under a condition that induced carbon catabolite repression: addition of $2 \%$ glucose $(17,18,26)$. Both the wild type and $\mathrm{AcOEC} 2$ were cultured for 10 days in liquid medium containing $1 \%(\mathrm{wt} / \mathrm{vol})$ pectin with or without $2 \%$ (wt/vol) glucose, and the transcript accumulation of AcCreA and Acpgl in the total RNA isolated from the mycelial mats as well as endoPG production in the culture filtrates were analyzed (Fig. 4). Transcription of Acpg1 and endoPG production of wild-type A. citri were repressed when $2 \%(\mathrm{wt} / \mathrm{vol})$ glucose was added to the pectin medium, as described previously $(17,18,26)$ (Fig. 4). However, transcripts of Acpgl were detected and endoPG also was produced in the culture filtrates from AcOEC2, and the repression observed in the wild type by addition of $2 \%$ (wt/vol) glucose was overcome by the AcCreA overexpression mutant AcOEC2 (Fig. 4). Transcription of $A c C r e A$ itself was independent of the addition of glucose, and the expression was not induced in either the wild type or AcOEC2 by glucose addition (Fig. 4A).

Symptom development in citrus due to AcCreA overexpression mutant. Spore suspensions $\left(1 \times 10^{5}\right.$ spores per $\left.100 \mu \mathrm{l}\right)$ of wild-type A. citri or the AcCreA overexpression mutant AcOEC2 were injected directly into the central axis of sterilized citrus fruit. After 2 weeks, the wild-type strain had not induced rotting except at the injection site, whereas AcOEC2 induced black rot symptoms in both the central axis and juice sac areas (Fig. 5). The wild-type strain also induced severe black rotting after 4 weeks, but the development of rotting was limited to the central axis area and the black rot symptom had not expanded into the juice sac area (Fig. 5). Unlike the typical symptoms caused by the wild type, AcOEC2 expressing AcCreA showed continuous expansion of black rot symptoms in both the central axis and juice sac areas (Fig. 5). Similar results were found in both the Washington navel orange and Hassaku orange.

\section{DISCUSSION}

Fungal CreA is a cis-acting zinc finger repressor protein involved in carbon catabolite repression. CreA is currently the only well-characterized factor involved in fungal carbon catabolite
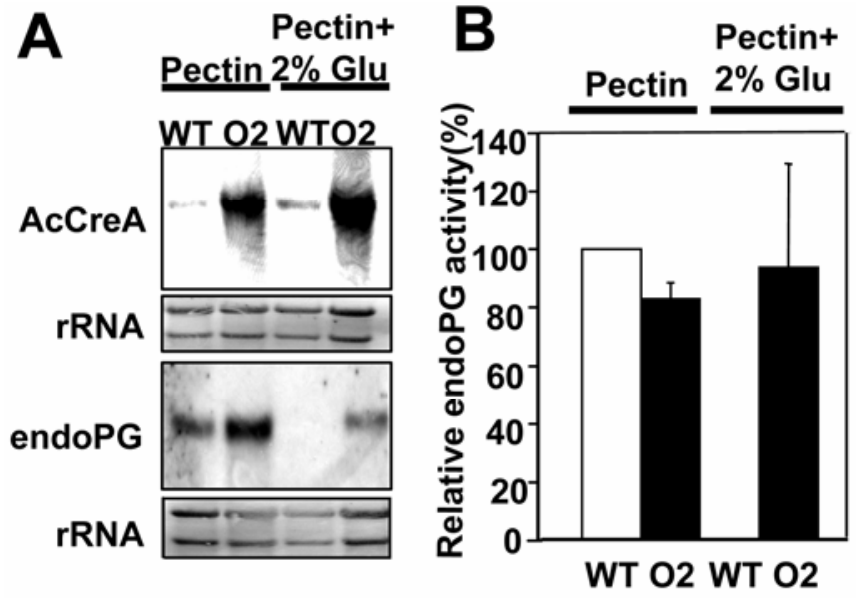

Fig. 4. Loss of carbon catabolite repression of endopolygaracturonase (endoPG) production in AcCreA overexpression mutant AcOEC2 of Alternaria citri. A, Northern blot analysis of AcCreA and endoPG genes in mycelial mats of 10-day-old cultures in pectin medium with or without addition of $2 \%$ (wt/vol) glucose. Equality of RNA loading in each lane was estimated by rRNA stained with methylene blue. B, Production of endoPG by AcOEC2 in the presence of glucose. PG activity was determined by a relative viscosity assay in 10-day-old culture filtrates of pectin medium with or without addition of $2 \%(\mathrm{wt} / \mathrm{vol})$ glucose from the A. citri wild type (WT) or AcCreA overexpression mutant AcOEC2 (O2). Activity of the wild type grown in pectin medium without glucose was counted as $100 \%$, and the relative percentage of each activity was calculated against it. Data indicate the mean of five independent repeats with standard deviation values. repression, but its role in the pathogenesis of fungal phytopathogens has never been examined. Some studies have reported the involvement of the carbon catabolite repressor, CreA, in the regulation of fungal-secreted cell wall-degrading enzymes $(2,11$, 22,27-29), and the overexpression of the creA gene resulted in a slightly higher repression of $\alpha$-amylase production in Aspergillus nidulans (1). Furthermore, mutation of creA in $A$. nidulans resulted in considerably elevated levels of $x \ln B$ mRNA (28). However, none of these studies linked the functions of CreA with regulation of the pathogenicity of the producing fungus.

To examine the relationship between the CreA-mediated carbon catabolite repression and the pathogenesis of phytopathogenic fungi, we made an overexpression mutant of AcCreA instead of a disruption mutant of this gene in Alternaria citri because a genomic Southern analysis of AcCreA in A. citri indicated the presence of multiple copies of this gene and also because disruption of the gene encoding CreA in Aspergillus nidulans produced a partially lethal phenotype or showed some defects on its growth $(12,35)$. Any defects on the growth of microbe are not suitable for use in studies of plant-microbe interactions because the defects will complicate the evaluation of the changes observed in plantmicrobe interactions.

The product of this gene, AcCreA, is similar to other fungal CreAs, which have a signature zinc finger domain of the $\mathrm{Cys}_{2} \mathrm{His}_{2}$ type $(3,31)$, and is expected to bind to the promoter region of target genes and downregulate gene expression, affecting secondary metabolites similar to the functions of CreA in other fungi (44). However, overexpression of the AcCreA gene in Alternaria citri unexpectedly led to a loss of the carbon catabolite repression function in the regulation of Acpgl gene expression. Direct evidence of AcCreA binding to the Acpgl promoter was not obtained because quantitative expression using prokaryotic systems damaged Escherichia coli cells and did not reproduce this regulatory protein (data not shown); thus, regular binding experiments for a regulatory protein, such as a gel shift assay, were impossible to perform without a large amount of AcCreA protein. However, the promoter sequence of the Acpgl gene has five typical binding sites (5'-SYGGRG-3') for CreA $(10,13)$ in the 813 bp upstream of the translation start site, and various deletion experiments of the promoter region found that the loss of the CreA binding site at -79 resulted in a loss of carbon catabolite repression on the transcription initiation function of the Acpgl promoter, clearly indicating the involvement of $\mathrm{CreA}$ in the regulation of Acpg1 expression (26). Therefore, the effect of AcCreA on the endoPG of A. citri was monitored by both transcription of Acpgl and production of Acpg1 in culture filtrates. Unlike the repression by the wild type, transcription of Acpgl in the AcCreA overexpression mutant AcOEC2 was not repressed by the addition of glucose and, thus, the production of endoPG in the culture filtrates
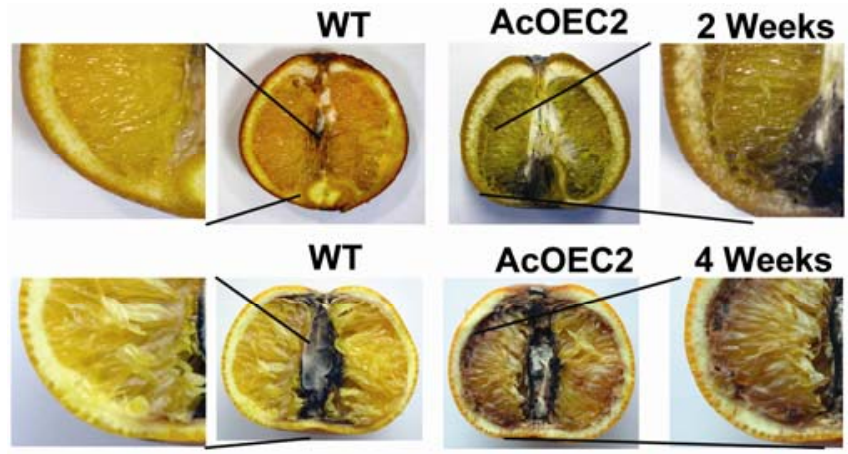

Fig. 5. Evaluation of virulence of $A c C r e A$ overexpression mutant AcOEC 2 of Alternaria citri. A spore suspension $\left(1 \times 10^{5}\right.$ spores $\left./ 100 \mu \mathrm{l}\right)$ of the wild type (WT) or AcCreA overexpression mutant (AcOEC2) was injected directly into the central axis of sterilized navel or Hassaku oranges. The inoculated fruit were incubated for 2 weeks (navel orange) or 4 weeks (Hassaku orange). 
also was not inhibited by carbon catabolite repression. Moreover, transcription of AcCreA itself was not significantly induced by addition of glucose, similar to results obtained for G. fujikuroi, B. cinerea, and $S$. sclerotiorum, in which creA transcription was independent of the addition of various carbon sources $(39,41)$. Under carbon catabolite repression, multiple genes encoding products that metabolize carbon sources other than glucose generally are known to be suppressed, but a relationship between the CreA-mediated catabolite repression and regulation of endoPG gene expression among fungal phytopathogens was reported only for $A$. citri and $S$. sclerotiorum $(26,29,41)$. CreA was suggested to be involved in the regulation of the $p g l$ gene from $S$. sclerotiorum by binding to the promoter sequence of $\mathrm{pg} 1$ (29), but the role of CreA in the pathogenesis of $S$. sclerotiorum was unknown.

The eradication of carbon catabolite repression by AcCreA overexpression that we found was directly linked to dramatic changes in symptom induction of $A$. citri in citrus fruit. AcOEC2 induced more severe symptoms at an earlier stage of infection, and rotting was observed in the juice sac area of citrus fruit, a symptom which the wild-type A. citri does not cause. As described earlier, black rot induction of this pathogen depends upon the production of an extracellular endoPG during the infection stage. After disruption of the Acpgl gene from A. citri (17), soft rot symptoms in the central axis area were reduced by $85 \%$ as a result of the inhibition of penetration and maceration of citrus tissue (17). Interestingly, the green fluorescence of A. citri transformant EPG7 carrying a GFP gene under control of the Acpg1 gene promoter of $A$. citri was induced by pectin in the peel during the infection stage, but repressed completely in the juice sac area, and the repression was expected to be caused by carbon catabolite repression by sugars in the juice (18). Our transformants containing constructs that fused CreA-binding site-deleted Acpg 1 promoters with the GFP reporter gene showed interesting phenotypes. Transformants with construct PGPDL4 with a -401 to -813 deletion had both substrate induction and catabolite repression, whereas transformants with PGPDL5 with an additional deletion from -1 to -84 , including one putative CreA-binding site, had substrate induction and loss of catabolite repression (26). Green fluorescence of transformants with PGPDL4 was induced in the peel by pectin but repressed completely in the juice sac area of citrus fruit, but transformants with PGPDL5 fluoresced green in both the peel and juice sac area, again indicating that the repression of A. citri endoPG gene of Acpgl in the juice sac area is under the regulation of carbon catabolite repression (26). AcOEC2 caused symptoms and rotting in the juice sac area, indicating that the repression of Acpgl expression in the wild type of $A$. citri in the juice sac area is the cause of catabolite repression and, thus, AcCreA-mediated catabolite repression controls the virulence or infection behaviors of this pathogen. As far as we know, this is the first example explaining the mechanism of the regulation of endoPG gene expression of phytopathogenic fungi in different tissues of host plants during different phases of infection by $\mathrm{CreA}$ and catabolite repression, as well as defining the relationship between pathogenesis and CreA or catabolite repression.

The mechanism causing the loss of carbon catabolite repression by overexpression of AcCreA is not clear at this point. However, an overdose of AcCreA to the promoter of Acpgl might act as an activator of Acpgl transcription instead of a repressor if the functional system of fungal CreA is similar to that of the yeast system. In yeast, the $\mathrm{Cys}_{2} \mathrm{His}_{2}$ zinc-finger-containing protein Mig1 binds to the promoters of several genes $(6,43)$ and causes carbon catabolite repression, similar to the function of filamentous fungal CreAs $(21,43)$. However, Mig1 has been known to recruit two other co-repressor proteins, Ssn6 and Tup1, and the complex of these three proteins inhibits the expression of genes encoding enzymes that catabolize other carbon sources $(38,43)$.
Ssn6 and Tup1 subunits are known to work as adaptor or effecter subunits, and are required for the repression function $(30,43)$. Interestingly, Mig1 alone worked as a transcriptional activator instead of a repressor when Ssn6 and Tup1 were absent $(38,43)$. If filamentous fungal CreA also includes a complex like the yeast Mig1 system for repression, overexpression of AcCreA might cause an imbalance in the subunit ratio and, thus, AcCreA alone caused activation the expression of Acpgl instead of repressing it. Identification of functional or sequence homologues of Ssn6 and Tup1 from filamentous fungi will verify our prediction, and their identification might explain the mechanism of the loss of carbon catabolite repression by overexpression of AcCreA.

\section{ACKNOWLEDGMENTS}

This study was supported in part by grants from the Ministry of Education, Culture, Sports, Science and Technology of Japan, the Japanese Society for the Promotion of Science, PROBRAIN, and PRESTO, Japan Science and Technology Agency. We thank T. Tsuge, Nagoya University, for providing transformation vector $\mathrm{pSH} 75$.

\section{LITERATURE CITED}

1. Agger, T., Petersen, J. B., O’Connor S. M., Murphy, R. L., Kelly, J. M., and Nielsen, J. 2002. Physiological characterization of recombinant Aspergillus nidulans strains with different creA genotypes expressing A. oryzae alpha-amylase. J. Biotechnol. 92:279-285.

2. Bautista, L. F., Aleksenko, A., Hentzer, M., Santerre-Henriksen, A., and Nielsen, J. 2000. Antisense silencing of the creA gene in Aspergillus nidulans. Appl. Environ. Microbiol. 66:4579-4581.

3. Berg, J. M. 1988. Proposed structure for the zinc-binding domains from transcription factor IIIA and related proteins. Proc. Natl. Acad. Sci. USA 85:99-102.

4. Brown, G. E., and Eckert, J. W. 2000. Alternaria rot. Page 37 in: Compendium of Citrus Diseases. The American Phytopathological Society, St. Paul, MN.

5. Brown, G. E., and MacCornack, A. A. 1972. Decay caused by Alternaria citri in Florida citrus fruit. Plant Dis. Rep. 56: 909-912.

6. Bu, Y., and Schmidt, M.C. 1998. Identification of cis-acting elements in the SUC2 promoter of Saccharomyces cereviseae required for activation of transcription. Nucleic Acids Res. 26:1002-1006.

7. Cooper, R. M. 1983. The mechanisms and significance of enzymic degradation of host cell walls by parasites. Pages 101-135 in: Biochemical Plant Pathology. J. A. Callow, ed. John Wiley \& Sons, New York

8. Cooper, R. M. 1984. The role of cell wall-degrading enzymes in infection and damages. Pages 13-27 in: Plant Diseases: Infection, Damage and Loss. R. K. S. Wood and G. J. Jellis, eds. Blackwell Scientific Publications, Oxford.

9. Cotty P. J., and Misaghi, I. J. 1984. Zinniol production by Alternaria species. Phytopathology 74:785-788.

10. Cubero, B., and Scazzocchio, C. 1994. Two different, adjacent and divergent zinc finger binding sites are necessary for CREA-mediated carbon catabolite repression in the proline gene cluster of Aspergillus nidulans. EMBO J. 13:407-415.

11. De Graaff, L. H., Van Den Broeck, H. C., Van Ooijen, A. J., and Visser, J. 1994. Regulation of the xylanase-encoding xlnA gene of Aspergillus tubigensis. Mol. Microbiol. 12:479-490.

12. Dowzer, C. E., and Kelly, J. M. 1991. Analysis of the creA gene, a regulator of carbon catabolite repression in Aspergillus nidulans. Mol. Cell Biol. 11:5701-5709.

13. Espeso, E. A., and Penalva, M. A. 1994. In vitro binding of the two-finger repressor $\mathrm{CreA}$ to several consensus and non-consensus sites at the ipnA upstream region is context dependent. FEBS Lett. 342:43-48.

14. Hicks, G. R., and Raikhel, N. V. 1995. Protein import into the nucleus: An integrated view. Annu. Rev. Cell Dev. Biol. 11:155-188.

15. Hofmann, K., Bucher, P., Falquet, L., and Bairoch, A. 1999. The PROSITE database, its status in 1999. Nucleic Acids Res. 27:215-219.

16. Isshiki, A., Akimitsu. K., Nishio, K., Tsukamoto. M., and Yamamoto, H. 1997. Purification and characterization of an endopolygalacturonase from the rough lemon pathotype of Alternaria alternata, the cause of citrus brown spot disease. Physiol. Mol. Plant Pathol. 51:155-167.

17. Isshiki, A., Akimitsu, K., Yamamoto, M., and Yamamoto, H. 2001. Endopolygalacturonase is essential for citrus black rot caused by Alternaria citri but not brown spot caused by Alternaria alternata. Mol. Plant-Microbe Interact. 14:749-757.

18. Isshiki, A., Ohtani, K., Kyo, M., Yamamoto, H., and Akimitsu, K. 2003. 
Green fluorescent detection of fungal colonization and endopolygalacturonase gene expression in the interaction of Alternaria citri with citrus. Phytopathology 93:768-773.

19. Kimura, N., and Tsuge, T. 1993. Gene cluster involved in melanin biosynthesis of the filamentous fungus Alternaria alternata. J. Bacteriol. 175:4427-4435.

20. Lu, S., Lyngholm, L., Yang, G., Bronson, C., Yoder, O. C., and Turgeon, B. G. 1994. Tagged mutations at the Tox1 locus of Cochliobolus heterostrophus by restriction enzyme-mediated integration. Proc. Natl. Acad. Sci. USA 91:12649-12653.

21. Lutfiyya, L. L., and Johnston, M. 1996. Two zinc-finger-containing repressors are responsible for glucose repression of SUC2 expression. Mol. Cell Biol. 16:4790-4797.

22. Mach, R. L., Strauss, J., Zeilinger, S., Schindler, M., and Kubicek, C. P. 1996. Carbon catabolite repression of xylanase I (xynl) gene expression in Trichoderma reesei. Mol. Microbiol. 21:1273-1281.

23. Masunaka, A., Tanaka, A., Tsuge, T., Peever, T. L., Timmer, L. W., Yamamoto, M., Yamamoto, H., and Akimitsu, K. 2000. Distribution and characterization of AKT homologs in the tangerine pathotype of Alternaria alternata. Phytopathology 90:762-768.

24. Mullaney, E. J., Hamer, J. E., Roberti, K. A., Yelton, M. N., and Timberlake, W. E. 1985. Primary structure of the $\operatorname{trp} C$ gene from Aspergillus nidulans. Mol. Gen. Genet. 199:37-45.

25. Nakai, K., and Kanehisa, M. 1991. Expert system for predicting protein localization sites in gram-negative bacteria. Proteins 11:95-110.

26. Ohtani, K., Isshiki, A., Katoh, H., Yamamoto, H., and Akimitsu, K. 2003. Involvement of carbon catabolite repression on regulation of endopolygalacturonase gene expression in citrus fruit. J. Gen. Plant Pathol. 69:120-125.

27. Orejas, M., MacCabe, A. P., Perez, G. J. A., Kumar, S., and Ramon, D. 1999. Carbon catabolite repression of the Aspergillus nidulans $x \ln A$ gene. Mol. Microbiol. 31:177-184.

28. Orejas, M., MacCabe, A. P., Perez G. J. A., Kumar, S., and Ramon, D. 2001. The wide-domain carbon catabolite repressor creA indirectly controls expression of the Aspergillus nidulans $x \ln B$ gene, encoding the acidic endo-(1,4)-xylanase X24. J. Bacteriol. 183:1517-1523.

29. Reymond-Cotton, P., Fraissinet-Tachet, L., and Fèvre, M. 1996. Expression of the Sclerotinia sclerotiorum polygalacturonase $p g 1$ gene: Possible involvement of CREA in glucose catabolite repression. Curr. Genet. 30:240-245.

30. Ronne, H. 1995. Glucose repression in fungi. Trends Genet. 11:12-17.

31. Rosenfeld, R., and Margalit, H. 1993. Zinc fingers: Conserved properties that can distinguish between spurious and actual DNA-binding motifs. J. Biomol. Struct. Dyn. 11:557-570.

32. Ruijter, G. J. G., and Visser, J. 1997. Carbon repression in Aspergilli. FEBS Lett. 151:103-114.

33. Sanger, F., Nickien, S., and Coulson, A. R. 1997. DNA sequencing with chain-terminating inhibitors. Proc. Natl. Acad. Sci. USA 74:5463-5467.

34. Schultz, J., Milpetz, F., Bork, P., and Ponting, C. P. 1998. SMART, a simple modular architecture research tool: Identification of signaling domains. Proc. Natl. Acad. Sci. USA 95:5857-5864.

35. Shroff, R. A., O'Connor, S. M., Hynes, M. J., Lockington, R. A., and Kelley, J. M. 1997. Null alleles of creA, the regulator of carbon catabolite repression in Aspergillus nidulans. Fungal Genet. Biol. 22:28-38.

36. Thompson, J. D., Higgins, D. G., and Gibson, T. J. 1994. CLUSTAL W: Improving the sensitivity of progressive multiple sequence alignment through sequence weighting, position-specific gap penalties and weight matrix choice. Nucleic Acids Res. 22:4673-4680.

37. Tonukari, N. J., Scott-Craig, J. S., and Walton, J. D. 2003. Isolation of the carbon catabolite repressor (CREA) gene from the plant-pathogenic fungus Cochliobolus carbonum. DNA Sequence 14:103-107.

38. Treitel, M. A., and Carlson, M. 1995. Repression by SSN6-TUP1 is directed by MIG1, a repressor/activator protein. Proc. Natl. Acad. Sci. USA 92:3132-3136.

39. Tudzynski, B., Liu, S., and Kelly, J. M. 2000. Carbon catabolite repression in plant pathogenic fungi: Isolation and characterization of the Gibberella fujikuroi and Botrytis cinerea creA genes. FEMS Microbiol. Lett. 184:9-15.

40. Vautard, G., Cotton, P., and Fèvre, M. 1999. The glucose repressor CRE1 from Sclerotinia sclerotiorum is functionally related to CREA from Aspergillus nidulans but not to the Mig proteins from Saccharomyces cerevisiae. FEBS Lett. 453:54-58.

41. Vautard-Mey. G., Cotton, P., and Fèvre, M. 1999. Expression and compartmentation of the glucose repressor CRE1 from the phytopathogenic fungus Sclerotinia sclerotiorum. Eur. J. Biochem. 266:252259.

42. Walton, J. D. 1994. Deconstructing the cell wall. Plant Physiol. 104:11131118.

43. Westergaard, S. L., Oliveira, A. P., Bro, C., Olsson, L., and Nielsen, J. 2007. A systems biology approach to study glucose repression in the yeast Saccharomyces cerevisiae. Biotechnol. Bioeng. 96:134-145. Published online. http://dx.doi.org/10.1002/bit.21135.

44. Yu, J.-H., and Keller, N. 2005. Regulation of second metabolism in fungi. Annu. Rev. Phytopathol. 43:437-458. 